\title{
Competitive Equilibrium and Stable Coalition in Overlay Environments
}

\author{
Shan Jiang ${ }^{1}$, Jianxin Liao ${ }^{1}$, Jun Gong ${ }^{1}$, Jingyu Wang ${ }^{1}$, Tonghong Li $^{2}$ \\ ${ }^{1}$ State Key Lab of Networking and Switching Technology \\ Beijing University of Posts and Telecommunications, Beijing, China \\ ${ }^{2}$ Department of Computer Science \\ Technical University of Madrid, Madrid, Spain \\ ${ }^{1}\left\{\right.$ jiangshan, liaojx, wangjingyu\}@bupt.edu.cn, ${ }^{1}$ gongjunonly@gmail.com, ${ }^{2}$ tonghong@fi.upm.es
}

\begin{abstract}
Overlay networks have been widely deployed upon the Internet to provide improved network services. However, the interaction between overlay and traffic engineering (TE) as well as among co-existing overlays may occur. In this paper, we adopt game theoretic approaches to analyze this hybrid interaction. Firstly, we model a situation of the hybrid interaction as an $\mathbf{n}+1$ player non-cooperative game, where overlays and TE are of equal status, and prove the existence of Nash equilibrium (NE). Secondly, we model another situation of the hybrid interaction as a 1-leadern-follower Stackelberg-Nash game, where TE is the leader and coexisting overlays are followers, and prove that the cost at Stackelberg-Nash equilibrium (SNE) is at least as good as that at NE for TE. Thirdly, we propose a cooperative coalition mechanism based on Shapley value to overcome the inherent inefficiency of $\mathrm{NE}$ and SNE, where players can improve their performance and form stable coalitions.
\end{abstract}

Keywords-overlay, traffic engineering, Nash equilibrium, Stackelberg game, coalition, Shapley value

\section{INTRODUCTION}

Overlays are logical networks built above the physical network, which can improve the network performance without modifying the underlay network. Over the past few years, a wide variety of overlay networks have been deployed upon the Internet by Service Providers (SPs) to provide different kinds of services, such as content delivery network (CDN), peer-to-peer network (P2P) and resilient overlay network (RON) [1]. Although these overlay applications improve the performance of traditional IP layer routing, the interaction between each overlay and underlay network, as well as the interaction among multiple co-existing overlay networks may occur. Internet Service Provider (ISP) adopts traffic engineering (TE) to optimize the global cost of the network. As the emerging overlays allocate traffic in the logical layer according to their own objectives, the established TE routing strategy may lead to sub-optimization for the underlay network. Thus, TE is triggered to readjust the routes and the new physical routes may turn back to affect the performance of overlays [2]-[6]. On the other hand, when multiple co-existing overlays are deployed upon the same physical network, their overlay routes may overlap each other since a physical link may belong to several overlay routes at the same time. These overlays compete for physical resources to optimize their own performance regardless of the impact on others, and they can interact with each other by adjusting the traffic on the overlapping routes [7]-[11]. For simplicity, we use hybrid interaction to represent the interaction between each overlay and TE and among co-existing overlays, which is shown in Fig. 1.

This paper studies the hybrid interaction of a scenario where multiple co-existing overlays are built upon the physical network of ISP. Since ISP provides the physical network for SPs in reality, its status should be equal or higher than SPs. Thus, we adopt two non-cooperative game models to analyze the hybrid interaction. We assume that the overlay's objective is to minimize its own delay cost and TE's objective is to minimize the congestion cost of the underlay physical network. In this paper, we make the following main contributions:

- We model a situation of the hybrid interaction as an $\mathrm{n}+1$-player non-cooperative game, where overlays and TE have equal status and the hybrid interaction between players ends up with a stable state that is Nash equilibrium (NE). We prove the existence of NE, which can be achieved through dynamic best response.

- We model another situation of the hybrid interaction as a 1-leader-n-follower Stackelberg-Nash game, where $\mathrm{TE}$ is the leader and overlays are followers. In this game, TE has higher status than overlays and plays its routing strategy first, and then all the overlays react optimally. We prove that the cost at Stackelberg-Nash equilibrium (SNE) is at least as good as that at NE for TE.

- In order to improve the performance of NE and SNE, we adopt a coalition game to explore a cooperative approach for co-existing overlays and TE. Our cooperative approach considers Pareto efficiency and fairness, where the players in the coalition cooperate to optimize the performance of the coalition and share costs based on Shapley value.

- Because of the NP-complexity of the problem, we apply distinct genetic algorithms (GA) to compute NE, SNE and the cost for each player in the coalition game.

\section{RELATED WORK}

Game theory [12] has been extensively used in networking research. A user equilibrium model for the interaction between network users as the standard network optimization problem was 


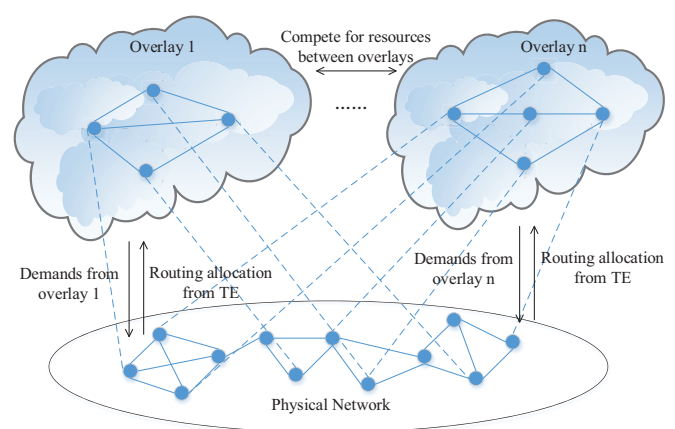

Fig. 1. Hybrid interaction in overlay environments

proposed by Roughgarden [13]. Liu et al. [2] studied the interaction between one single overlay and TE by using bestreply dynamics and demonstrated the impact of overlay routing on the underlay network. Wang et al. [3] studied the interaction between the P2P overlay and TE and pointed out the nonoptimal performance of the network. Jiang et al. [7] studied the interaction between multiple co-existing overlays on top of a physical network and proved that the interaction may cause the efficiency loss and fairness paradox in multiple overlay routing. Keralapura et al. [8] studied the interaction among co-existing overlays competing for limited network resources. Xiao et al. [6] modeled the interaction of overlays and underlay networks in multi-domain networks as a congestion game and provided some operational guidelines to ensure system stability.

Researchers have explored some ways to solve the conflicts in the interaction. Jiang et al. [7] proposed a pricing scheme to improve the performance of overlays. Gong et al. [4] adopted a repeated game to reduce the oscillations between overlay and TE. Cohen et al. [14] studied the optimization problem of deploying overlay nodes. Wang et al. [10] studied the collaborations of multiple selfish overlays by using multi-path resources. Yang et al. [11] studied the interaction among multiple co-existing P2P systems and proposed an ISP-friendly inter-overlay coordination framework to control P2P traffic. Cooperative game theory can be applied as an alternative way to overcome the inefficiency of NE. Jiang et al. [5] and Cui et al. [9] adopted a Nash bargaining theory to improve the inefficiency of NE. However, Nash bargaining can only be applied to the game with two players. When there are more players in the game, the problem becomes more complex. Ma et al. [15] applied Shapley value to network environments for ISP settlement. Niyato et al. [16] considered a mobile cloud computing environment in which cooperative SPs can form a coalition to create a resource pool to support the mobile applications and share the revenue obtained by the resource pool. Misra et al. [17] proposed an ideal incentive structure based on Shapley cooperative theory so that each content provider can receive a fair price for the usage of its resources.

\section{Model And Problem Statement}

In this section we model the co-existing overlays and TE.

\section{A. Network Model}

Let $G=(V, E)$ represent the underlay network, where $V$ is the set of physical nodes and $E$ is the set of links. Then we define a capacity vector $C=\left(c_{e_{1}}, c_{e_{2}}, \cdots, c_{e_{|E|}}\right)$, where $c_{e}$ is the capacity for each link $e \in E$, a routing set $R$, where each route $r \in R$ denotes a possible route of the underlay network, and a $|E| \times|R|$ physical indicator matrix $A$ where $a_{e r}=1$ if route $r$ traverses link $e$, and $a_{e r}=0$ otherwise.

An overlay $s$ in the logical level is represented by graph $G^{(s)}=\left(V^{(s)}, E^{(s)}\right)$, where $V^{(s)}$ is the set of logical nodes and $E^{(s)}$ is the set of logical links. Each overlay node maps to a physical node, and each overlay link maps to a set of physical routes, i.e. $r \rightarrow e^{(s)}$. A logical path $r^{(s)} \in R^{(s)}$ contains a set of logical links. Each overlay may have several demands, each of which is a source-sink pair associated with a flow $f$ with traffic volume $w_{f}$. Consider there are $n$ overlays on top of an underlay and let $N$ denote the full set of overlays. Let $F_{S}$ denote all demands of overlay $s$. Also, we consider background demands from underlay users that directly use the underlay network to transfer data. Let $F_{b}$ represent all background demands. We use set $F=\cup_{s \in N} F_{s} \cup F_{b}$ to denote all flows. Let $b^{(i, f)}, i \in N, b$ denote a $\left|E^{(i)}\right| \times\left|R_{f}^{(i)}\right|$ logical indicator matrix, where $b_{e r}^{(i, f)}=$ 1 if flow $f$ traverses overlay link $e^{(i)}$, and $b_{e r}^{(i, f)}=0$ otherwise. Then we rewrite the matrix $B$ as: $B=$ $\left(b^{(1)}, \cdots, b^{(n)}, b^{(b)}\right)^{T}, b^{(i)}=\left(b^{\left(i, f_{1}\right)}, \cdots, b^{\left(i, f_{\left|F_{i}\right|}\right)}\right) i \in N, b$.

The overlay determines the routing of all demands for its overlay users. For each flow $f \in F_{s}$, the overlay needs to decide how to assign its traffic $w_{f}$ to possible routes. Thus, we can define an allocation decision vector for flow $y^{(s, f)}=$ $\left(y_{r_{1}^{(s)}}^{(s, f)}, y_{r_{2}^{(s)}}^{(s, f)}, \cdots, y_{\left|R_{f}^{(s)}\right|}^{(s, f)}\right)^{T}$, where $y_{r^{(s)}}^{(s, f)}$ is the traffic volume on logical path $r^{(s)}$ for flow $f$ in overlay $s$. We have $\sum_{r^{(s)} \in R_{f}^{(s)}} y_{r^{(s)}}^{(s, f)}=w_{f}$. Similarly, $y^{(b, f)}$ is defined for each background flow $f \in F_{b}$, as no routing policy can be applied for those background flows, thus $y^{(b, f)}=w_{f}$. We then write: $Y=$ $\left(y^{(1)}, \cdots, y^{(n)}, y^{(b)}\right), y^{(i)}=\left(y^{\left(i, f_{1}\right)}, \cdots, y^{\left(i, f_{\left|F_{i}\right|}\right)}\right)^{T} i \in N, b$.

The overlay and background users pass on their demands to underlay. And TE decides how to allocate the traffic on physical links. The total volume between two neighbor logical nodes in fact maps a physical demand, i.e., $\sum_{i \in N, b} e^{(i)}$ corresponds to demands from the logical source node of $e^{(i)}$ to the sink node. Denote $X$ as a $|R| \times \sum_{i \in N, b}\left|E^{(i)}\right|$ matrix and its element $x_{r e^{(i)}}$ is the fraction of volume from logical links that TE allocates to route $r$, and we have $\sum_{r \in R_{e}(i)} x_{r e^{(i)}}=1$ for each flow logical link. Here TE does not differentiate demands between overlay and underlay users and performs the same fractions for demands with the same source-sink. Then the volume on each link $e: L=$ $\left(l_{e_{1}}, l_{e_{2}}, \cdots l_{e_{|E|}}\right)^{T}$ is:

$$
\begin{gathered}
L=A X \sum_{i \in N, b} \sum_{f \in F} b^{(i, f)} y^{(i, f)}=A X \sum_{i \in N, b} b^{(i)} y^{(i)} \\
=A X B Y,
\end{gathered}
$$

where $l_{e}$ is the volume of demands allocated to physical link $e$.

We say allocation decisions $X, Y$ of overlay and TE are feasible if they satisfy the conditions that $Y \geq 0, X \geq 0, L^{T} \leq C$. i.e., the volume on logical links and fraction on physical links are non-negative and the aggregate volume allocated to link $e$ is no more than its capacity $c_{e}$. 


\section{B. Objective of TE}

The objective of TE is to minimize the congestion cost in the whole physical network. Denote $o_{e}\left(l_{e}\right)$ as the congestion function for physical link $e \in E$, and the congestion function is continuous, increasing and convex. For all physical links, we define $O(L)=\left(o_{e_{1}}\left(l_{e_{1}}\right), o_{e_{2}}\left(l_{e_{2}}\right), \cdots, o_{e_{|E|}}\left(l_{e_{|E|}}\right)\right)^{T}, e \in E \quad$ as the congestion function. Let $f(X)$ denote the congestion cost of the whole physical network. Therefore:

$$
f(X)=\sum_{e \in E} o_{e}\left(l_{e}\right) .
$$

The optimization problem for TE can be rewritten as:

$$
\begin{aligned}
& \min f(X)=\delta O(L) \\
& \text { s.t. }\left\{\begin{array}{l}
\forall e^{(s)} \in E^{(s)}, \sum_{r \in R_{e}(s)} x_{r e^{(s)}}=1, \\
Y \geq 0, X \geq 0, L^{T} \leq C
\end{array}\right.
\end{aligned}
$$

where $\delta=(1,1, \cdots, 1),|\delta|=|E|$.

\section{Objective of Overlays}

The objective of overlay routing is to minimize the delay in its overlay network. Here, we adopt an additive link delay cost, where the path delay is the summation of delay costs of all links along this path and the flow delay is the weighted summation of path delay used by this flow. Denote $d_{e}\left(l_{e}\right)$ as the unit delay function of physical link $e \in E$ and the delay function is continuous, increasing and convex. For all physical links $e \in E$, we define the unit delay function $D(L)=$ $\left(d_{e_{1}}\left(l_{e_{1}}\right), d_{e_{2}}\left(l_{e_{2}}\right), \cdots, d_{e_{|E|}}\left(l_{e_{|E|}}\right)\right)^{T}$. Let $g^{(s)}\left(y^{(s)}\right)$ denote the delay for overlay $s$. By using the notations above, the delay for overlay $s$ is:

$$
g^{(s)}\left(y^{(s)}\right)=\sum_{e^{(s)} \in E^{(s)}} l_{e^{(s)}} \sum_{e \in r, r \rightarrow e^{(s)}} x_{r e^{(s)}} d_{e}\left(l_{e}\right),
$$

where $l_{e^{(s)}}$ represents the volume on logical link $e^{(s)} \in E^{(s)}$.

Here we expand the size of $b^{(s)}$ and $y^{(s)}$ to $B$ and $Y$, respectively, by filling the vacant elements with zero. The optimization problem for overlay $s$ can be rewritten as:

$$
\begin{aligned}
& \min g^{(s)}\left(y^{(s)}\right)=\left(A X b^{(s)} y^{(s)}\right)^{T} D(L) \\
& \text { s.t. }\left\{\begin{array}{l}
\forall f \in F_{s}, \sum_{r^{(s)} \in R_{f}^{(s)}} y_{r^{(s)}}^{(s, f)}=w_{f}, \\
Y \geq 0, X \geq 0, L^{T} \leq C
\end{array}\right.
\end{aligned}
$$

where $y^{(-s)}$ is the allocation from other overlays.

\section{N+1-PLAYER NON-COOPERATIVE GAME}

In this section, we consider a situation of the hybrid interaction where co-existing overlays and TE have equal status and model it as an $\mathrm{n}+1$-player non-cooperative game.

\section{A. Non-cooperative Game}

We define a set of players $N+1=\{1,2, \cdots, n+1\}$, where first $n$ players are overlays and the last is TE. The set of overlay $s$ is a volume allocation matrix of demands and the set of TE is a fraction allocation matrix of flows. The set is:

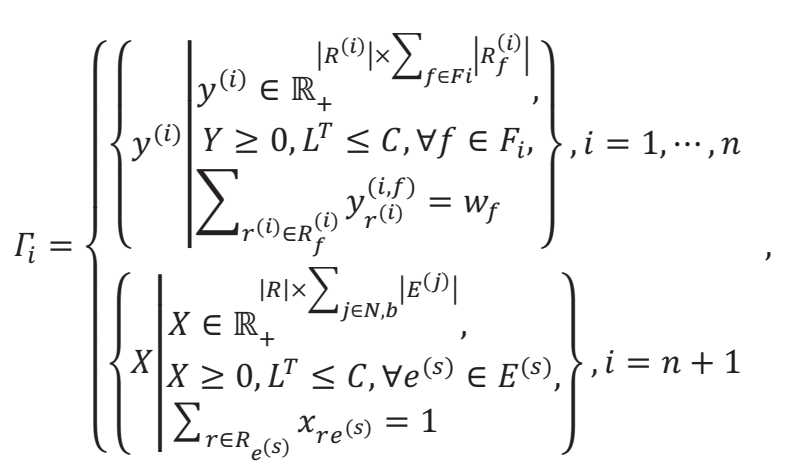

where $\mathbb{R}_{+}$is the non-negative set. Furthermore, let $U_{s}$ denote the payoff function of overlay $s$ with $U_{i}=$ $-g^{(i)}\left(y^{(i)}\right), i=1,2, \cdots, n$. Let $U_{n+1}$ denote the payoff function of TE with $U_{n+1}=-f(X)$. Finally, we define the $\mathrm{n}+1$-player non-cooperative game $G\langle N+1, \Gamma, U\rangle$, where $\Gamma=\Gamma_{1} \times \ldots \times$ $\Gamma_{n} \times \Gamma_{n+1}$ denotes the set of strategy profiles and $U=U_{1} \times \ldots \times$ $U_{n} \times U_{n+1}$ denotes the set of corresponding utility profiles. Then we have the following definition of NE, which describes a situation where no player can improve its own objective by altering its routing strategy unilaterally.

Definition 1. A feasible strategy profile $\left(Y^{*}, X^{*}\right) \in \Gamma$, $\left(Y^{*}, X^{*}\right)=\left(y^{*(1)}, y^{*(2)}, \cdots, y^{*(n)}, X^{*}\right)$ is NE if for each overlay $i \in N$ and TE:

$\forall y^{(i)} \in \Gamma_{i}$,

$U_{i}^{*}=-g^{(i)}\left(y^{*(i)}, y^{*(-i)}, X^{*}\right) \geq-g^{(i)}\left({y^{\prime}}^{(i)}, y^{*(-i)}, X^{*}\right)$.

$\forall X^{\prime} \in \Gamma_{n+1}$,

$U_{n+1}^{*}=-f\left(Y^{*}, X^{*}\right) \geq-f\left(Y^{*}, X^{\prime}\right)$

Theorem 1. In $G\langle N+1, \Gamma, U\rangle$, NE exists if $g^{(s)}$ and $f$ functions are continuous, increasing and convex.

Proof: If NE exists, the game should meet two conditions [18]: (1) Each player's strategy space $\Gamma_{i}$ is a nonempty compact convex subset of a Euclidean space. (2) The preference relation between $U$ is quasi-concave and continuous on $\Gamma_{i}$. Firstly, the strategy spaces in $G\langle N+1, \Gamma, U\rangle$ are well defined by the capacity of links and the non-negativity constraint $Y \geq 0, X \geq$ $0, L^{T} \leq C$ with a closed and bounded feasible region, thus $\Gamma_{i}$ is compact. Moreover, all constraints are affine functions and the feasible domain is the intersection of half-spaces and hyperplanes, thus $\Gamma_{i}$ is convex. Hence, $G\langle N+1, \Gamma, U\rangle$ meets the first condition. Secondly, $g^{(s)}$ and $f$ are continuous and convex, hence, the payoff functions $U_{s}$ and $U_{n+1}$ are continuous and quasi-concave on $\Gamma_{i}$ and so is preference relation between $U$. Therefore, $G\langle N+1, \Gamma, U\rangle$ meets the second condition.

In order to compute the NE allocation for overlays and TE, we first define the notation of best response. And NE is the status where each player adopts its best response.

Definition 2. In a game $G\langle N+1, \Gamma, U\rangle$, each player's best response to the strategies of other players is the strategy that minimizes its objective function, which is:

$$
\begin{aligned}
& y^{(i)}\left(y^{(-i)}, X\right)=\arg \min g^{(i)}\left(y^{(i)}, y^{(-i)}, X\right), i=1, \cdots n \\
& X(Y)=\arg \min f(X, Y), i=n+1
\end{aligned} .
$$




\section{B. Genetic Algorithm}

Since solving optimal allocations for TE and overlays is NPhard, we apply genetic algorithm (GA) [19] to obtain the solution, which is algorithm 1 .

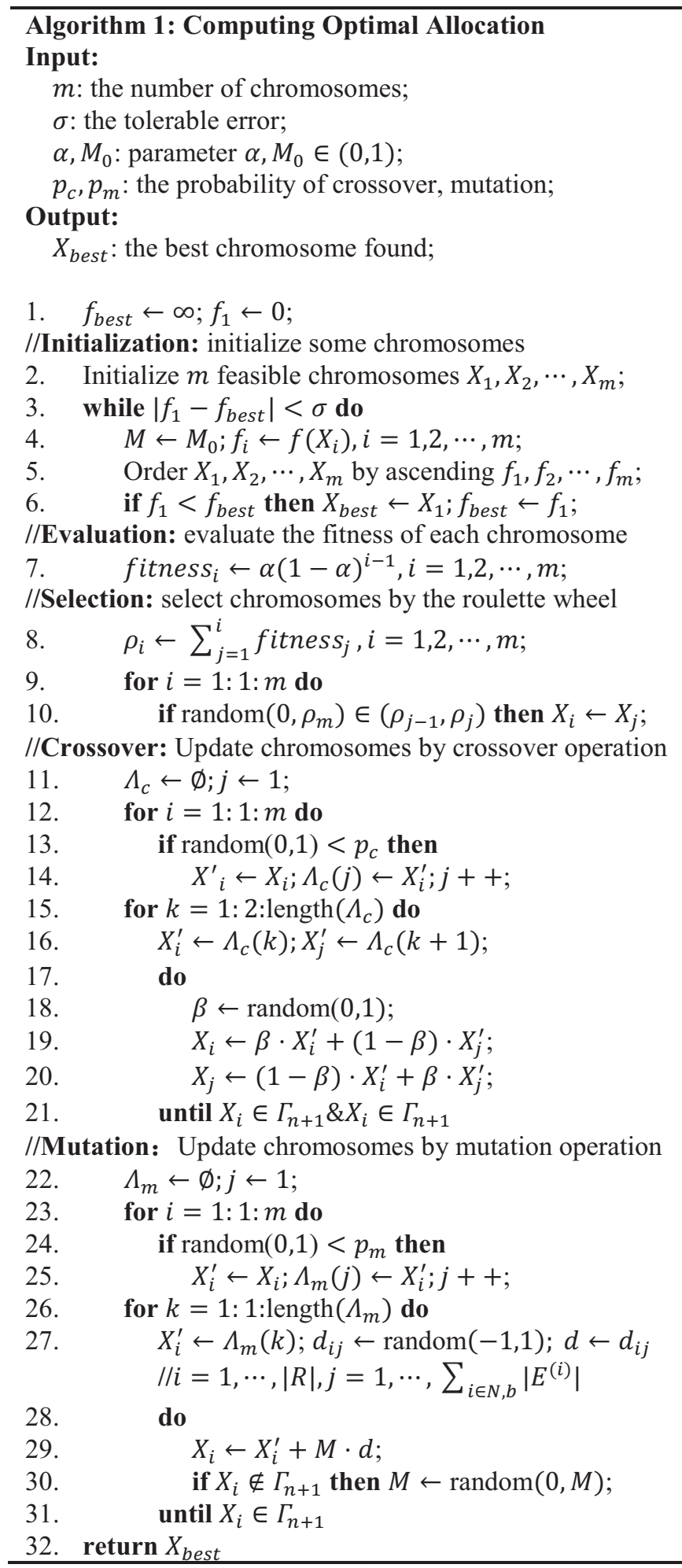

Similarly, we can use algorithm 1 to compute the dynamic best response for each overlay $s$ by replacing $X_{i}, X_{\text {best }}, f_{i}, f_{\text {best }}$ with $y_{i}^{(s)}, y_{\text {best }}^{(s)}, g_{i}^{(s)}, g_{\text {best }}^{(s)}$.

\section{Dynamic Best Response}

In general, players do not possess perfect information of other players at the beginning of the game, and they can only play their strategies based on the existent situation. Nevertheless, since players repeatedly interact with each other, they will gradually obtain the information and finally have the perfect information, which leads to the convergence of NE. In order to compute NE, we first give TE and overlay networks an initial allocation, which is an arbitrary feasible allocation of $X, Y$. Then, TE and overlays take turns to use algorithm 1 to compute their dynamic best responses until they reach NE, whchi is algorithm 2 .

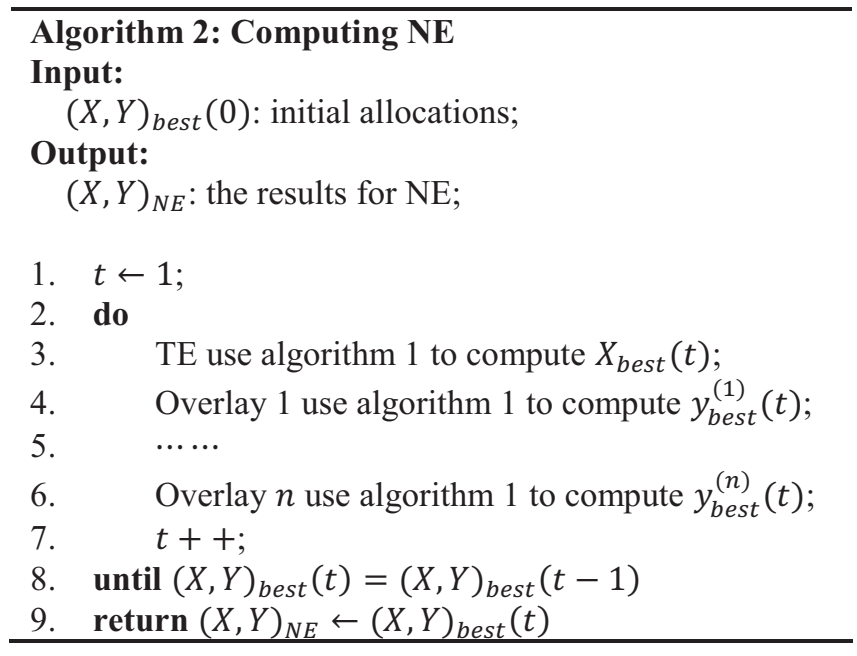

\section{1-LEADER-N-FOLLOWER STACKELBERG-NASH GAME}

In this section, we consider a situation where TE has higher status than all overlays. We model this interaction as a 1-leader$\mathrm{n}$-follower Stackelberg-Nash game [19], where TE is the leader with complete knowledge of strategies of all the following overlays.

\section{A. Stackelberg-Nash Equilibrium}

For each routing strategy $X \in \Gamma_{n+1}$ of TE as the leader, let $Y(X)$ denote the reaction set of overlays dependent on $X$ such that $Y(X)=\left\{Y \mid Y \in \Gamma_{1} \times \Gamma_{2} \times \cdots \times \Gamma_{n}\right\}$. Assume that TE first chooses its routing strategy $X \in \Gamma_{n+1}$ as the leader, and then all overlays determine their strategies $\left(y^{(1)}, y^{(2)}, \cdots, y^{(n)}\right) \in Y(X)$ The problem of the 1-leader-n-follower Stackelberg-Nash game can be described as:

$$
\begin{aligned}
& \min f(X, Y(X)) \\
& \text { s.t. } X \in \Gamma_{n+1} \\
& \text { where } y^{(1)}, y^{(2)}, \cdots, y^{(n)} \text { solves:. } \\
& \quad \min g^{(s)}\left(y^{(s)}, y^{(-s)}, X\right) \\
& \quad \text { s.t. } y^{(s)} \in \Gamma_{s}
\end{aligned}
$$

This optimization problem is classified as bi-level programming (BP) problem [20]. In this Stackelberg-Nash game, all overlays are of equal status. For all overlays, the best solution is the NE among them, which is defined by $Y^{*}(X)=$ $\left(y^{*(1)}, y^{*(2)}, \cdots, y^{*(n)}\right) \in Y(X)$ with respect to $X$. Then, we have the following definition of SNE for the 1-leader-n-follower Stackelberg-Nash game. 
Definition 3. A feasible strategy profile $\left(Y^{*}\left(X^{*}\right), X^{*}\right) \in \Gamma$, $\left(Y^{*}\left(X^{*}\right), X^{*}\right)=\left(y^{*(1)}, y^{*(2)}, \cdots, y^{*(n)}, X^{*}\right)$ is SNE if and only if:

$$
\begin{aligned}
& \forall X^{\prime} \in \Gamma_{n+1}, Y^{*}\left(X^{\prime}\right)=\left(y^{\prime(1)}, y^{(2)}, \cdots, y^{\prime(n)}\right) . \\
& U_{n+1}^{*}=-f\left(X^{*}, Y^{*}\left(X^{*}\right)\right) \geq-f\left(X^{\prime}, Y^{*}\left(X^{\prime}\right)\right)
\end{aligned} .
$$

According to this definition, SNE prescribes an optimal strategy for TE, if TE plays first and then all overlays react optimally. We can easily prove that the cost at SNE is at least as good as that at NE for TE.

Theorem 2. In $G\langle N+1, \Gamma, U\rangle$, the cost at SNE is at least as good as that at NE for TE.

Proof: Let $(\bar{Y}, \bar{X})$ be the NE and $\left(Y^{*}\left(X^{*}\right), X^{*}\right)$ be the SNE. If TE first choose $\bar{X}$ as the leader, $Y^{*}(\bar{X})=\bar{Y}$ holds for all overlays as followers. Thus, we have $\bar{U}_{n+1}=-f(\bar{X}, \bar{Y})=-f\left(\bar{X}, Y^{*}(\bar{X})\right)$. By the definition of SNE, we have $\bar{U}_{n+1}=-f\left(\bar{X}, Y^{*}(\bar{X})\right) \leq$ $U_{n+1}^{*}=-f\left(X^{*}, Y^{*}\left(X^{*}\right)\right)$.

\section{B. Algorithm for Solving Stackelberg-Nash Equilibrium}

For the BP problem described in (9), we also use GA to search the optimal strategy for TE. The following algorithm 3 is proposed to compute SNE:

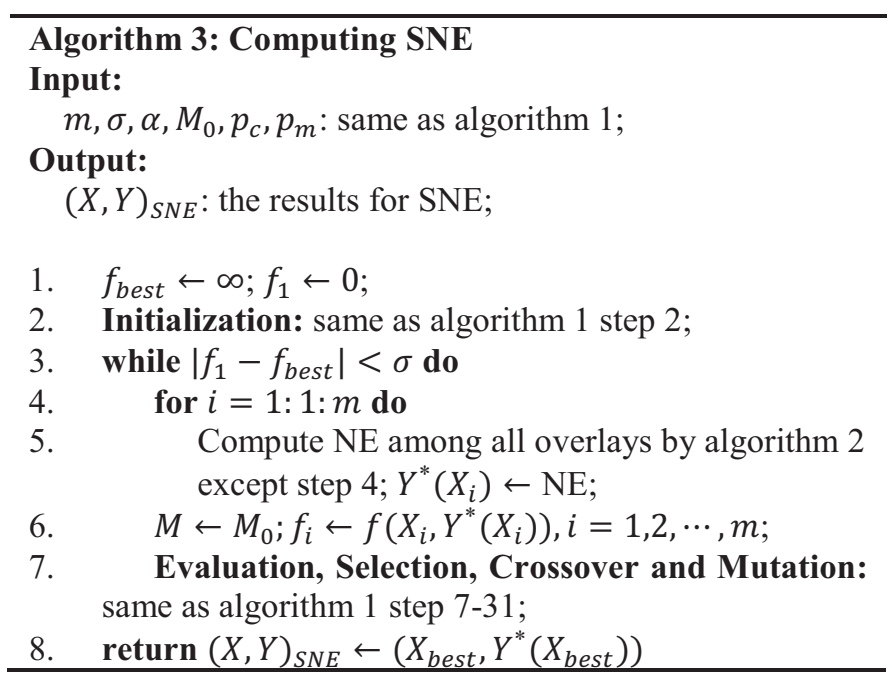

\section{CoAlition Game}

It has been pointed out that NE and SNE in the noncooperative game are usually inefficient [13]. In order to improve the performance of overlays and TE at NE and SNE, we propose a cooperative coalition mechanism by applying the concepts of core and Shapley value to determine the share of cost taken by each player.

\section{A. Coalition Game}

A coalition game [12] is denoted by $\langle\mathbb{C}, v\rangle$, where $\mathbb{C} \subseteq N+$ 1 is the coalition representing the set of cooperative players in the same group and $v(\cdot)$ is the coalitional function for coalition $\mathbb{C}$. $\mathbb{C}=N+1$ is called the grand coalition. There could be multiple coalitions and the set of all coalitions is referred to as coalitional structure $\Phi=\left\{\mathbb{C}_{1}, \mathbb{C}_{2}, \cdots, \mathbb{C}_{|\Phi|}\right\}$ such that $N+1=$ $\cup_{i=1}^{|\Phi|} \mathbb{C}_{i}, \mathbb{C}_{i} \cap \mathbb{C}_{j}=\varnothing$ and $\mathbb{C}_{i} \subseteq \Phi$. The objective value $v(\mathbb{C})$ for the coalition $\mathbb{C}$ is defined by the unified cost of all players in the coalition, and players in the same coalition will cooperate to minimize the objective cost of the whole coalition. Thus, $v(\mathbb{C})$ is defined by:

$$
\begin{aligned}
& v(\mathbb{C})=\min \sum_{i \in \mathbb{C}} \tau_{i} \\
& \text { where } \tau_{i}=\left\{\begin{array}{l}
g^{(i)}\left(y^{(i)}, y^{(-i)}\right), i=1,2, \cdots, n, \\
\alpha f(X), i=n+1
\end{array}\right.
\end{aligned}
$$

where $\alpha$ is the equivalent weight between congestion and delay, i.e., when sharing cost, 1 unit congestion equals to $\alpha$ units delay. The value of $\alpha$ is depended on the negotiation results of TE and overlays or other external factors. Thus, the interaction between coalitions is a non-cooperative game and will end up with NE after several iterations. Likewise, we use algorithm 2 to compute $\mathrm{NE}$ for the interaction between coalitions regarding each coalition as a player in the game.

\section{1) Core Solution}

We first define the core among cooperative players in coalition $\mathbb{C}$, which is:

core $=\left\{z \mid \forall S \subseteq \mathbb{C}, \sum_{i \in \mathbb{C}} z_{i}=v(\mathbb{C}), \sum_{i \in S} z_{i} \leq v(S)\right\}$,

where $z=\left(z_{i}, i \in S\right)$, and $z_{i}$ is the assigned cost for player $i$ in coalition $\mathbb{C}$. The core is a set of cost shares which guarantee that no player will leave the coalition $\mathbb{C}$ and form subcoalition $S \subseteq$ $\mathbb{C}$. Namely, the summation of assigned costs by coalition $\mathbb{C}$ is always less than or equal to that of assigned costs by any subcoalition $S$ (i.e., $\sum_{i \in S} z_{i} \leq v(S)$ ). Thus, the core solution stabilizes the coalition.

\section{2) Shapley Value}

We now apply the concept of Shapley value to assign fair cost shares to each player in coalition $\mathbb{C}$. The Shapley value for player $i$ in coalition $\mathbb{C}$ can be obtained as follows:

$$
\varphi_{i}(v)=\sum_{S \subseteq \mathbb{C} \backslash\{i\}} \frac{|S| !(|\mathbb{C}|-|S|-1) !}{|\mathbb{C}| !}[v(S \cup i)-v(S)]
$$

The Shapley value $\varphi_{i}(v)$ is obtained by evaluating the contribution of each player $i$ in reducing the cost of the coalition, which determines the cost to be shared by player $i$. The Shapley value is suitable for cost share of the cooperative players in coalition $\mathbb{C}$ because of the following properties.

Efficiency: Since $\sum_{i \in \mathbb{C}} \varphi_{i}(v)=v(\mathbb{C})$, the summation of costs of all cooperative players is minimized.

Symmetry: For two arbitrary player $i, j \in \mathbb{C}$, if $v(S \cup i)=v(S \cup$ $j$ ) holds for all the subcoalition $S \subseteq \mathbb{C}$ without these two players, then $\varphi_{i}(v)=\varphi_{j}(v)$. That is, when players $i$ and $j$ have the same contribution to the coalition, the cost shares of the players $i$ and $j$ will be equal.

Dummy: For a player $i$, if $v(S)=v(S \cup i)$ holds for all the subcoalition $S \subseteq \mathbb{C}$ without player $i$, then $\varphi_{i}(v)=0$. That is, if player $i$ does not contribute to the total cost of the coalition (e.g., overlay $i$ has no traffic in the network), then cost share of this player will be zero.

Additivity: If $v$ and $v^{\prime}$ are the coalitional functions, then $\varphi(v+$ $\left.v^{\prime}\right)=\varphi\left(v^{\prime}+v\right)=\varphi(v)+\varphi\left(v^{\prime}\right)$. 
For the Shapley value, the individual efficiency and fairness can be achieved. Specifically, the cost shared by the cooperative player is less than or equal to the cost of the player having no cooperation with other players $\left(\varphi_{i}(v) \leq v(i)\right)$. Moreover, the Shapley value is unique.

\section{B. Coalition Formation}

We assume that all players are rational and self-interested to minimize their own costs by forming a coalition. The coalition formation process can be described as a non-cooperative game. The set of players consisting of all overlays and TE is $N+1$. In the game, each player has to decide whether to form coalition with other players. The cooperation between player $i$ and player $j$ can be denoted by a binary variable $q_{i j}$, where $q_{i j}=1$ if they cooperate and $q_{i j}=0$ otherwise. The strategy of player $i$ is $q_{i}=\left(q_{i 1}, q_{i 2}, \cdots, q_{i, n+1}\right)$. Thus we rewrite the strategies of all players $Q$ as $Q=\left(q_{1}, q_{2}, \cdots, q_{n+1}\right) \quad, \quad q_{i j}=q_{j i}, i, j=$ $1,2, \cdots, n+1$. The feasible set of strategies of each player is described as follows:

$\Omega_{i}$

$=\left\{q_{i} \mid \begin{array}{l}q_{i j} \in\{0,1\}, j \in N+1 \\ q_{i j}=\left\{\begin{array}{l}1, \text { if } i, j \in \mathbb{C}, \forall \mathbb{C} \\ 0, \text { if } i \notin \mathbb{C} \text { or } j \notin \mathbb{C}, \forall \mathbb{C}\end{array}, i=1, \cdots, n+1\right.\end{array}\right\}$.

The NE $Q^{*}=\left(q_{1}^{*}, q_{2}^{*}, \cdots, q_{n+1}^{*}\right)$ for the coalition formation game can be defined as follows:

$\forall i \in N+1, q_{i}^{\prime} \in \Omega_{i}, \varphi_{i}\left(q_{i}^{*}, q_{-i}^{*}\right) \leq \varphi_{i}\left(q_{i}^{\prime}, q_{-i}^{*}\right)$.

The NE of the coalition formation among players can be obtained from the algorithm based on the dynamic best response. The player will make a decision on cooperation formation iteratively. In each iteration, the player evaluates the new strategy, and then switches to the new strategy achieving the least cost. The algorithm to compute NE for coalition formation game is demonstrated as follows:

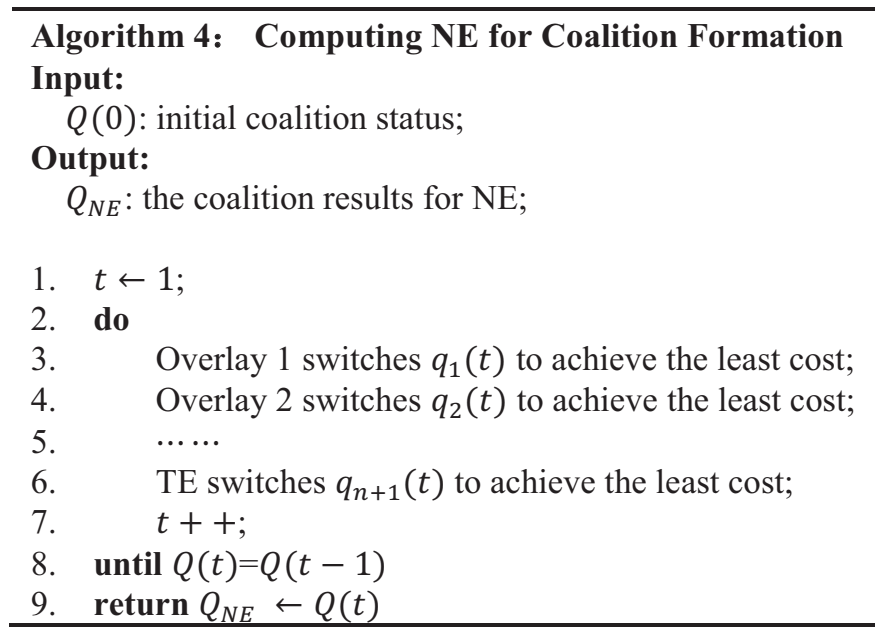

\section{SimUlation AND EVALUATION RESUltS}

In this section, we conduct simulations to evaluate the difference of performances of two situations of the hybrid interaction, and then compare them with the performance in coalition game.

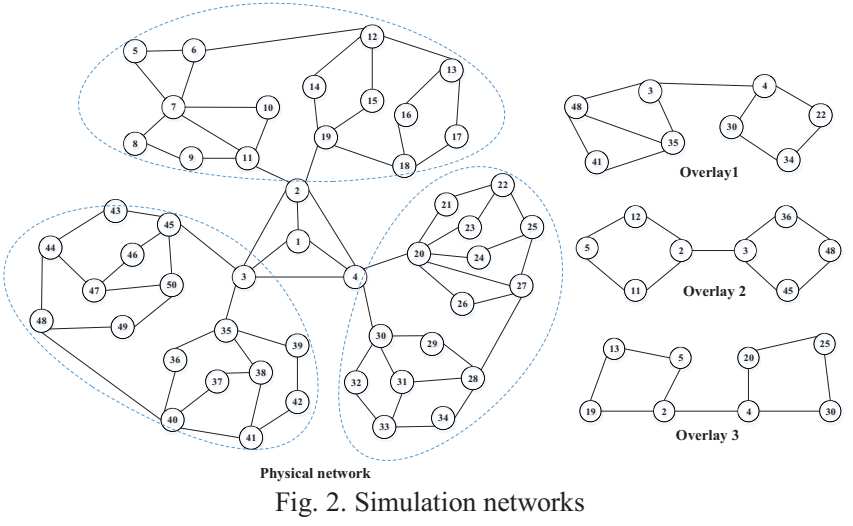

A. Simulation Setup

We use a 50-node underlay network with three co-existing overlays deployed above sharing some common physical links and nodes, which is shown in Fig. 2. The underlay network consists of one central region and three marginal regions. For each overlay, there is one flow pair with demand $w_{f}=1$. Besides, there is one unit background demand between every neighbor overlay nodes. In order to simulate the situation such that overlays compete for the limited common link bandwidth, we set the capacity of central links as $c_{e}=3$ and the capacity of marginal links as $c_{e}=7$, respectively.

The delay function $d_{e}\left(l_{e}\right)$ and congestion function $o_{e}\left(l_{e}\right)$ for a physical link are chosen as follows. First, the link delay $d_{e}\left(l_{e}\right)$ generally consists of queuing delay and propagation delay such that $d_{e}\left(l_{e}\right)=1 /\left(c_{e}-l_{e}\right)+p$, where the queuing delay is approximated by the $\mathrm{M} / \mathrm{M} / 1 \operatorname{model} 1 /\left(c_{e}-l_{e}\right)$ and the propagation delay is equal to a constant value $p$. We set the value $p$ as one in our simulation. Second, the link congestion $o_{e}\left(l_{e}\right)$ is modeled as a piecewise linear, increasing and convex function, which is described as follows [5]:

$$
\begin{aligned}
& o_{e}\left(l_{e}\right) \\
& =\left\{\begin{array}{lr}
l_{e}, & 0 \leq l_{e} / c_{e}<1 / 3 \\
3 l_{e}-2 / 3 c_{e}, & 1 / 3 \leq l_{e} / c_{e}<2 / 3 \\
10 l_{e}-16 / 3 c_{e}, & 2 / 3 \leq l_{e} / c_{e}<9 / 10 \\
70 l_{e}-178 / 3 c_{e}, & 9 / 10 \leq l_{e} / c_{e}<1 \\
500 l_{e}-1468 / 3 c_{e}, & 1 \leq l_{e} / c_{e}<11 / 10 \\
5000 l_{e}-16318 / 3 c_{e}, 11 / 10 \leq l_{e} / c_{e}<\infty
\end{array}\right.
\end{aligned}
$$

The input parameters we set in algorithm 1 are: $m=50$, $\sigma=0.001, \alpha=0.5$, and $M_{0}=0.1$. Furthermore, we set the probability of crossover as $p_{c}=0.8$ and the probability of mutation as $p_{m}=0.5$.

\section{B. Simulation Results}

Our simulation results demonstrate the efficiency loss caused by the hybrid interaction in the network, as well as the variation of routing decisions during the interaction process.

\section{1) Nash Equilibrium}

In this simulation, the sequence of interactions executed is TE-overlay1-overlay2-overlay3. In the beginning, there are only background demands in the underlay network for TE and then overlay 1, 2, 3 start to transfer their data in turn. In each iteration, each player applies algorithm 1 to optimize its strategy. In order 
to reduce the iteration time of the algorithm, we set some initial chromosomes such that TE adopts the shortest route, and set others as arbitrary feasible allocation.

The simulation results are shown in Fig. 3 and Fig. 4. We observe sudden increases of congestion and delay at the beginning because the traffic demands increase when overlay 1 , 2, 3 start to transfer their data. Then we observe some oscillations in the middle of the process caused by the conflicts between overlays and TE. Eventually, the oscillations subside and come to a stable state, which is the NE and the results of NE are: $f=126.2054, g^{(1)}=11.6089, g^{(2)}=11.2442, g^{(3)}=$ 11.2983. The simulation results demonstrate how dynamic best responses converge to the NE. However, in general, the results may vary due to the multiplicity of NE. Nevertheless, the convergence is inevitable after certain iterations, which leads to a stable NE.

\section{2) Stackelberg-Nash Equilibrium}

In this simulation, we use the results of NE from the previous simulation as initial chromosomes to search SNE for TE. We choose the value of least congestion cost equal to that at SNE for TE. In this simulation, we obtain a SNE: $f=126.1532, g^{(1)}=$ $11.8404, g^{(2)}=11.2394, g^{(3)}=11.0585$. The simulation results demonstrate that $\mathrm{TE}$ can obtain less congestion cost at SNE than at NE. Fig. 5 shows the interaction process of overlays when TE first plays the strategy at SNE. We observe that the interaction among overlays converge to a stable state after several iterations.

\section{3) Stable Coalition Solution}

In this simulation, we evaluate the coalition formation of three overlays (denoted by OR1, OR2 and OR3) and TE with $\alpha=1$. Table 1 shows the Shapley value obtained by each player with different coalition formations. There are totally 15 coalition structures. We can apply algorithm 4 to reach the stable coalition. We observe that the stable coalition structure is $\Phi_{12}^{*}$, where TE, overlay 1 and overlay 3 cooperate, and overlay 2 is separate. $\Phi_{12}^{*}$ is stable since all players have no better choice than staying in their current coalitions. Note that $\Phi_{1}$ is the situation of $n+1$-player non-cooperative game. $\Phi_{15}$ is the situation of global optimal routing, which achieves the least total cost of all players. However, if TE is concerned about its own cost, it will leave $\Phi_{15}$ and go to $\Phi_{14}$. And then, overlays take turns to reconsider their strategies. The convergence path of coalition formation is $\Phi_{15} \rightarrow \Phi_{14} \rightarrow \Phi_{10} \rightarrow \Phi_{11} \rightarrow \Phi_{12}^{*}$.

\section{4) Performance Comparison}

We compare the cost of TE and overlays, which is demonstrated in Fig. 6. We observe that TE and all overlays can improve their performance through cooperation. Moreover, the volume of cost they reduce is assigned fairly by Shapley value. Thus, the simulation results provide an effective cooperative solution for both ISP and SPs.

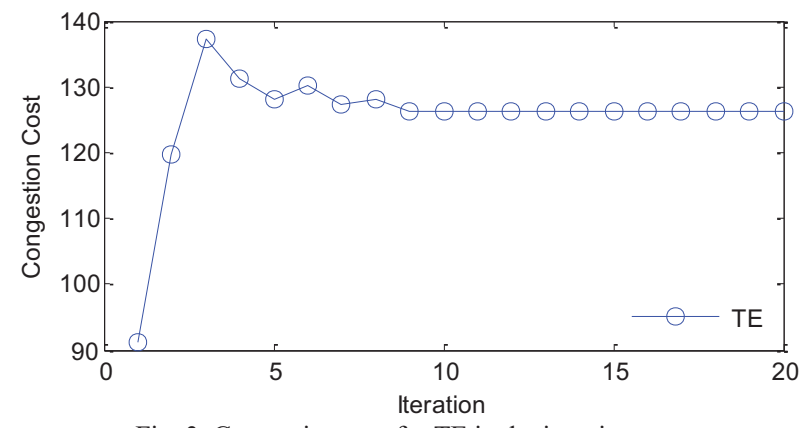

Fig. 3. Congestion cost for TE in the iteration process

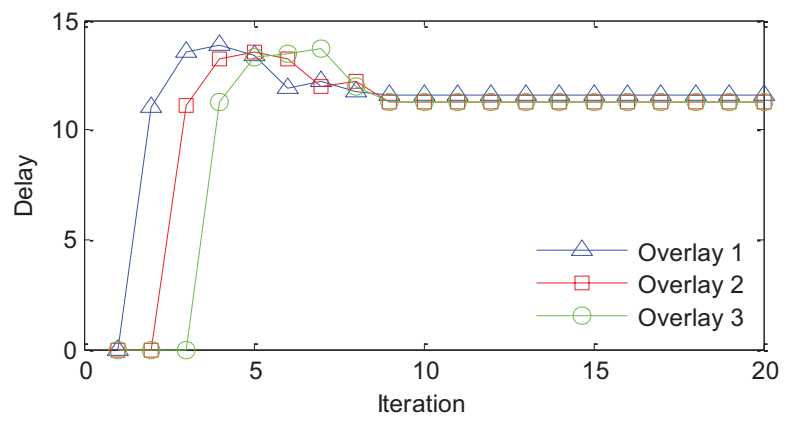

Fig. 4. Delay for three overlays in the iteration process

TABLE I. COST ALlocation BASED on the Shapley VAlue

\begin{tabular}{|c|c|c|c|c|}
\hline \multirow{2}{*}{ Coalition structure } & \multicolumn{4}{|c|}{ The Shapley Value } \\
\cline { 2 - 5 } & TE & OR1 & OR2 & OR3 \\
\hline$\Phi_{1}=\{\{\mathrm{TE}\},\{\mathrm{OR} 1\},\{\mathrm{OR} 2\},\{\mathrm{OR} 3\}\}$ & 126.2054 & 11.6089 & 11.2442 & 11.2983 \\
\hline$\Phi_{2}=\{\{\mathrm{TE}, \mathrm{OR} 1\},\{\mathrm{OR} 2\},\{\mathrm{OR} 3\}\}$ & 125.2191 & 10.6226 & 11.1827 & 11.1705 \\
\hline$\Phi_{3}=\{\{\mathrm{TE}, \mathrm{OR} 1\},\{\mathrm{OR} 2, \mathrm{OR} 3\}\}$ & 124.6511 & 10.9444 & 11.2185 & 11.2062 \\
\hline$\Phi_{4}=\{\{\mathrm{TE}, \mathrm{OR} 2\},\{\mathrm{OR} 1\},\{\mathrm{OR} 3\}\}$ & 125.7572 & 11.5884 & 10.7967 & 11.3351 \\
\hline$\Phi_{5}=\{\{\mathrm{TE}, \mathrm{OR} 2\},\{\mathrm{OR} 1, \mathrm{OR} 3\}\}$ & 125.0102 & 11.6381 & 11.0724 & 11.3847 \\
\hline$\Phi_{6}=\{\{\mathrm{TE}, \mathrm{OR} 3\},\{\mathrm{OR} 1\},\{\mathrm{OR} 2\}\}$ & 125.8066 & 11.5962 & 11.1864 & 10.8995 \\
\hline$\Phi_{7}=\{\{\mathrm{TE}, \mathrm{OR} 3\},\{\mathrm{OR} 1, \mathrm{OR} 2\}\}$ & 125.1411 & 11.5058 & 11.0959 & 11.1833 \\
\hline$\Phi_{8}=\{\{\mathrm{OR} 1, \mathrm{OR} 2\},\{\mathrm{TE}\},\{\mathrm{OR} 3\}\}$ & 125.2908 & 11.5721 & 11.2074 & 11.3333 \\
\hline$\Phi_{9}=\{\{\mathrm{OR} 1, \mathrm{OR} 3\},\{\mathrm{TE}\},\{\mathrm{OR} 2\}\}$ & 125.1300 & 11.5767 & 11.1922 & 11.2667 \\
\hline$\Phi_{10}=\{\{\mathrm{OR} 2, \mathrm{OR} 3\},\{\mathrm{TE}\},\{\mathrm{OR} 1\}\}$ & 125.2707 & 11.5635 & 11.2002 & 11.2543 \\
\hline$\Phi_{11}=\{\{\mathrm{TE}, \mathrm{OR} 1, \mathrm{OR} 2\},\{\mathrm{OR} 3\}\}$ & 125.0268 & 10.8417 & 11.0151 & 11.1728 \\
\hline $\boldsymbol{\Phi}_{\mathbf{1 2}}^{*}=\{\{\mathrm{TE}, \mathrm{OR} 1, \mathrm{OR} 3\},\{\mathrm{OR} 2\}\}$ & $\mathbf{1 2 4 . 8 5 5 9} *$ & $\mathbf{1 0 . 6 2 6 2} *$ & $\mathbf{1 1 . 1 8 2 3} *$ & $\mathbf{1 0 . 9 0 2 9} *$ \\
\hline$\Phi_{13}=\{\{\mathrm{TE}, \mathrm{OR} 2, \mathrm{OR} 3\},\{\mathrm{OR} 1\}\}$ & 125.5609 & 11.5516 & 10.9544 & 11.0579 \\
\hline$\Phi_{14}=\{\{\mathrm{OR} 1, \mathrm{OR} 2, \mathrm{OR} 3\},\{\mathrm{TE}\}\}$ & 124.2889 & 11.6234 & 11.2469 & 11.3056 \\
\hline$\Phi_{15}=\{\{\mathrm{TE}, \mathrm{OR} 1, \mathrm{OR} 2, \mathrm{OR} 3\}\}$ & 124.6839 & 10.7464 & 11.0749 & 10.9627 \\
\hline
\end{tabular}




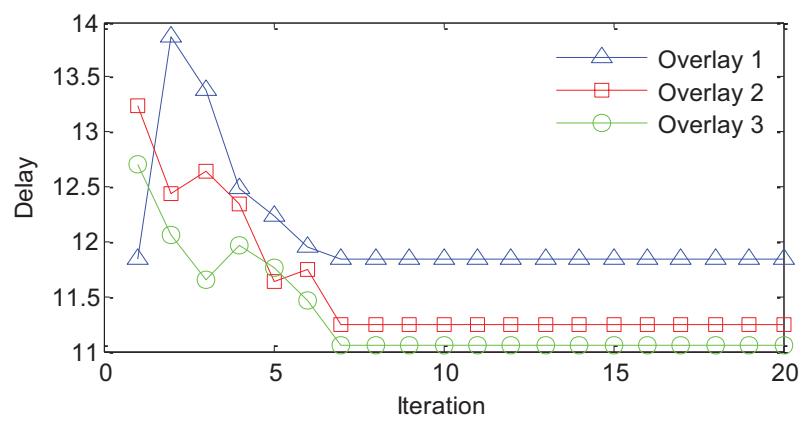

Fig. 5. Delay for three overlays when TE plays SNE strategy
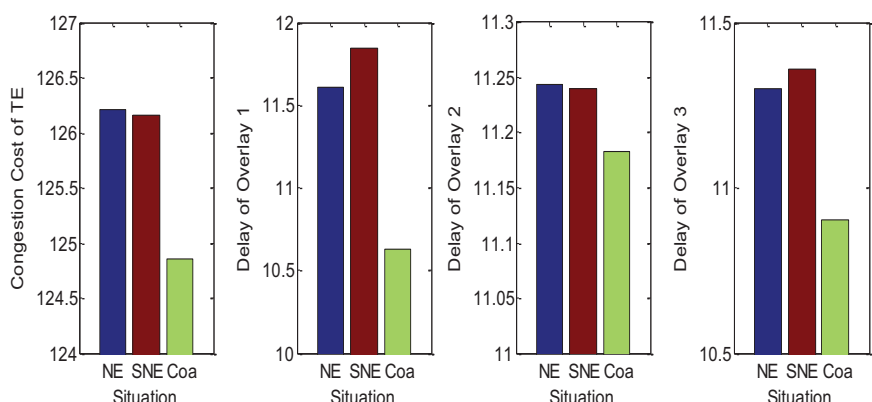

Fig. 6. Cost comparison of NE, SNE, Coalition

\section{CONCLUSION}

This paper focuses on the scenario where multiple coexisting overlays are deployed above a physical network. We consider two situations of the hybrid interaction, and model them as an $n+1$-player non-cooperative game and a 1-leader-nfollower Stackelberg-Nash game, respectively. However, the results for overlays and TE at NE and SNE are inefficient. In order to improve the performance of $\mathrm{NE}$ and $\mathrm{SNE}$, we propose a cooperative coalition game based on Shapley value. We observe that the performance of all overlays and TE is significantly improved by the stabilized coalition game. However, the delay and congestion cost is inherent non-transferable, the Shapley value needs external coordination, therefore, the approach to reduce negotiation costs and to specify equivalent weight $\alpha$ is worthy of future study.

\section{ACKNOWLEDGMENT}

This work was jointly supported by: (1) the National Basic Research Program of China (No. 2013CB329102); (2) National Natural Science Foundation of China (No. 61471063, 61421061, 61372120 , 61271019, 61101119, 61121001); (3) the Key (Keygrant) Project of Chinese Ministry of Education. (No. MCM20130310); (4) Beijing Municipal Natural Science Foundation (No. 4152039); (5) Beijing Higher Education Young Elite Teacher Project (No. YETP0473); (6) Spanish Research Council (No: TIN2013-46883); (7) Regional Government of Madrid (No: S2013/ICE-2894) cofunded by FSE \& FEDER.

\section{REFERENCES}

[1] D. Andersen, H. Balakrishnan, F. Kaashoek, R. Morris, "Resilient overlay networks," in Proc. of the 18th ACM Symposium on Operating Systems Principles (SOSP), pp.131-145, Oct. 21-24, 2001.
[2] Y. Liu, H. Zhang, W. Gong, and D. Towsley, "On the interaction between overlay routing and underlay routing," in Proc. of the 24th Annual Joint Conference of the IEEE Computer and Communications Societies (INFOCOM), pp. 2543-2553, Mar. 13-17, 2005.

[3] C. Wang, N. Wang, M. Howarth, G. Pavlou, "On the interactions between non-cooperative P2P overlay and traffic engineering behaviors," in Proc. of the 53th IEEE Global Telecommunications Conference (GLOBECOM), pp. 1-6, Dec. 6-10, 2010.

[4] J. Gong, J. Liao, J. Wang, Q. Qi, L. Zhang, "Reducing the oscillations between overlay routing and traffic engineering by repeated game theory," in Proc. of the 19th Asia-Pacific Conference on Communications(APCC), pp.591-596, 2013.

[5] W. Jiang, R. Zhang, J. Rexford, M. Chiang, "Cooperative content distribution and traffic engineering in an ISP network," ACM SIGMETRICS Performance Evaluation Review, vol.37, no.1, pp.239-250, 2009.

[6] J. Xiao, R. Boutaba, "Reconciling the overlay and underlay tussle," IEEE/ACM Transactions on Networking (TON), vol.22, no.5, pp.1489-1502, 2014.

[7] W. Jiang, D. M. Chiu, and J. C. S. Lui, "On the interaction of multiple overlay routing," Performance Evaluation, pp. 229-246, Oct. 2005.

[8] R. Keralapura, C. N. Chuah, N. Taft, G. Iannaccone, "Race conditions in coexisting overlay networks," IEEE/ACM Transactions on Networking (TON), vol.16, no.1, pp.1-14, 2008.

[9] Z. Cui, J. Liao, J. Wang, Q. Qi, J. Wang, "Cooperative overlay routing in a multiple overlay environment," in Proc. of the IEEE International Conference on Communications (ICC), pp.31003105, 2014.

[10] J. Wang, J. Liao, T. Li, J. Wang, "On the collaborations of multiple selfish overlays using multi-path resources," Peer-toPeer Networking and Applications, vol.8, no.2, pp.203-215, 2014.

[11] P. Yang, L. Xu, "An ISP-friendly inter-overlay coordination framework for multiple coexisting P2P systems," Peer-to-Peer Networking and Applications, vol.7, no.4, pp.396-409, 2014.

[12] M. Osborne, A. Rubinstein, A course in game theory. MIT press, 1994.

[13] T. Roughgarden and E. Tardos, "How bad is selfish routing?," Journal of the ACM, vol.49, no.2, pp.236-259, 2002.

[14] R. Cohen, D. Raz, "Cost-effective resource allocation of overlay routing relay nodes," IEEE/ACM Transactions on Networking (TON), vol.22, no.2, pp.636-646, 2014.

[15] R. T. B. Ma, D. M. Chiu, J. C. S. Lui, V. Misra, D. Rubenstein, "Internet economics: the use of Shapley value for ISP settlement," IEEE/ACM Transactions on Networking (TON), vol.18, no.3, pp.775-787, 2010.

[16] D. Niyato, P. Wang, E. Hossain, W. Saad, Z. Han, "Game theoretic modeling of cooperation among service providers in mobile cloud computing environments," in Proc. of the IEEE Wireless Communications and Networking Conference (WCNC), pp.3128-3133, Apr.1-4, 2012.

[17] V. Misra, S. Ioannidis, A. Chaintreau, L. Massouli, "Incentivizing peer-assisted services: a fluid Shapley value approach," in Proc. of the ACM SIGMETRICS International Conference on Measurement and Modeling of Computer Systems, pp.215-226, June14-18, 2010.

[18] J. B. Rosen, "Existence and uniqueness of equilibrium points for concave n-person games," Econometrica: Journal of the Econometric Society, vol.33, no.3, pp.520-534, 1965.

[19] B. Liu, "Stackelberg-Nash equilibrium for multilevel programming with multiple followers using genetic algorithms," Computers \& Mathematics with Applications, vol.36, no.7, pp.79-89, 1998.

[20] L. Vicente, P. Calamai, "Bilevel and multilevel programming: a bibliography review," Journal of Global Optimization, vol.5, no.3, pp.291-306, 1994. 